\title{
Effects of Auditory Brainstem Response (ABR) on Measuring Children's Auditory Functions: An Experimental Investigation
}

\author{
Sadeq Ali Saad Al Yaari (Corresponding author) \\ Independent Researcher, Dept. of English, College of Arts, King Saud University (KSU) \\ Riyadh, Kingdom of Saudi Arabia \\ E-mail: prof.sadeq@gmail.com
}

\begin{abstract}
Nassr Almaflehi
A professor of Statistics, College of Applied Medical Sciences, King Saud University (KSU), Riyadh, Kingdom of Saudi Arabia

E-mail: nassr_almaflehi@hotmail.com
\end{abstract}

Received: October 28, 2013 Accepted: November 10, 2013 Published: December 12, 2013

doi:10.5296/jsel.v1i2.4735 URL: http://dx.doi.org/10.5296/jsel.v1i2.4735

\begin{abstract}
Background: Measuring hearing functional capabilities by Auditory Brainstem Responses (ABR) may contribute to better treatment, and possible differences in this process may have important clinical implications.
\end{abstract}

Objectives: To measure the validity and reliability of ABR through screening, estimating, and intraoperative monitoring auditory capabilities of Arab infants and children and the degree of their seriousness.

Design: Pre-and-posttest was administered to measure the validity and reliability of ABR.

Settings: Al Khars hospital in Al Ahsa'a, Kingdom of Saudi Arabia (KSA).

Participants: Subjects of the present study are sixty (60) individuals. The study classified them into two groups: Infants $(\mathrm{N}=30$, ages range between $0-40$ weeks $)$ and children $(\mathrm{N}=30$, ages range between 10 months -3 years), diagnosed with auditory problems.

Procedures: ABR pre-and-posttest measurement was administered during two weeks. The 
outcomes of the pre-and-posttest were neuropsycholinguistically and statistically analyzed.

Main outcomes \& results: The results of the pre-and-posttest for both infants and children did not vary significantly. Also consistent with expectations, higher scores were not registered for the infants' measurements due to age factors. The findings from this study largely indicate that $\mathrm{ABR}$ is valid and reliable.

Keywords: Auditory, Brainstem, Response, Children, Measurement, Function, Experimental study

\section{Introduction Chapter}

\subsection{Introduction}

In the past three decades, auditory brainstem response (ABR) studies have revealed that auditory brainstem evoked responses measured in newborns may predict delayed or impaired development. For example, Murray (1988)'s study attempted to determine the degree to which these auditory brainstem evoked responses could be measured during the first year of life in 93 normal and abnormal infants. The researcher investigates whether knowledge of abnormalities in the newborn ABR gives us more information about infant's eventual developmental outcome than simply knowing the child initial risk status. According to Murray, "To address this question, group assignment (low risk or high-risk) was coded as a dummy valuable and used as a covariate in analyses of variance of the effects of ABR diagnosis on the dependent measures of outcome" (Murray, 1988: 1547).

The researcher concludes that abnormalities in the newborn ABR may indicate diffuse central nervous system dysfunction in addition to deficits specific to the auditory system. Such findings inspired other neuropsycholinguistic researchers to conduct many studies to investigate the distinctive features of the ABR. The purpose was to examine to what extent ABR could be used to examine hearing of children. Uzuka, et al., (1996)'s study examined ABR threshold to see whether or not it is more effective than to pursue the trends in each component of ABR. In the study, tone burst sound stimuli were employed and ABR threshold shift caused by kanamycin administration was investigated in dogs. In a series of monitoring of ABR against short-period auditory lesions, changes in ABR waveform after intravenous administration of kanamycin were detected. The results found in the study suggest that tracing of ABR threshold by tone bursts could provide information that has specificity for frequency in hearing tests and is a useful method in clinical veterinary medicine and/ or toxicological tests.

Neuropsycholinguistically, one of the functions of ABR is to measure the degree of the hearing loss in children who suffer from hearing disorders. Thus, when an infant or a child has auditory deficit, the first assessment tool neuropsycholinguists prescribe for him is ABR. The purpose of Kawarai, et al., (1999)'s study was to statistically examine the reliability of ABR for the diagnosis of profound deafness in early childhood according to our experience over the past 12 years of follow-up in our facility. Subjects of the study included 371 children 
among 1,041 children who were admitted to Kanariya-Gakuen (institute for pre-school deaf children) from April 1985 to March 1997. Results indicate that in five cases with an abnormal hearing threshold determined by ABR and other audio-logical tests, repeated examinations carried out during a 5- to 6-month follow-up period revealed that they had normal hearing. Three of these children had been diagnosed previously with mental retardation and the remaining two were infants. Additionally, the specificity and sensitivity of ABR were calculated as $97.3 \%$ and $100 \%$, respectively. The predictive value of a positive result was $94.7 \%$. There remains the possibility of a false negative for such cases, although the rate seems to be very low $(0.2 \%)$. Commenting on the predictive value of the negative results, the research team made it clear that it was $100 \%$. The research team has rightly stated:

"Predictive value of negative results by ABR in children's hearing impairment was $100 \%$ in this study. It was shown that unusual results of ABR could have predicted hearing impairment, at least to a higher degree than any other available audio-logical examination." (Kawarai, et al., 1999: p.9)

Early ABR sets the stage for a lifetime of competence in hearing capacities. However, the objective and behavioral mechanisms associated with the relative advantages and disadvantages of both ABR and behavioral thresholds are not well explored. In Garnham, et al., (2000)'s study, a comparative study between unaided and aided hearing thresholds, by both objective and behavioral techniques was held. Twenty (20) children (aged $<1-10$ years) whose parents reported that they developed hearing loss were evaluated with standardized measures of ABR. Sequencing ability to measure children with hearing disorders is recognized as a fundamental component in ABR. It is claimed that gap threshold based on ABR would be similar to the psychophysical gap detection threshold. Sequencing relationships between the measurements results of the two techniques are studied (Werner, et al., 2001). Outlined results reveal that psychophysical gap detection thresholds of 3- and 6-month-old infants were an order of magnitude worse than those of adults with normal hearing, as previously reported; however, ABR gap thresholds of 3-month-old infants were no different from those of adults with normal hearing. These results, according to Wener and his co-workers suggest that ABR gap thresholds and psychophysical gap detection depend on at least some of the same mechanisms within the auditory system.

Such results like those mentioned earlier encouraged researchers to develop new ideas for assessment tools. New patterns of assessment tools were differently investigated by many neuropsycholinguists in the field. In Purdy's study (2002), for example, the researcher reports patterns of assessment tools, attempting to determine whether tone bursts gated on and off using a nonlinear, exact-Blackman-gating function would be a more frequency-specific stimulus for auditory brain stem response audiometry than the more traditional 2-1-2 cycle linearly gated tone burst. In terms of age differences due to that ABR's studies are always conducted on children, tone burst ABRs were recorded in 10 adults with normal hearing and in 18 adults with sloping high-frequency sensori-neural hearing loss. The researcher concludes that there are no clear differences between Blackman- and linearly gated tone bursts in terms of how well ABR thresholds predicted pure-tone thresholds at 2000 and 4000 Hz. In general, audiometric thresholds were predicted with good accuracy $(+/-15 \mathrm{~dB})$ by the 
tone burst ABR thresholds. The $4000-\mathrm{Hz}$ audiometric threshold was underestimated in one subject with a very steeply sloping hearing loss by both Blackman- and linearly gated tone burst ABR thresholds, indicating that ipsilateral masking such as notched noise would be needed to ensure frequency specificity in this and similar cases.

The generator of $\mathrm{ABR}$ during development through $\mathrm{ABR}$ and focal superior olivary complex recordings were measured to examine age-related differences in hearing processing. Although neuropsycholinguists appear to be familiar with human outcomes, notably children as regards to many ABR measurements and experiments, little is known about ABR's results in non-human. The effect of ABR measurements on assessing animals' auditory performance was investigated using twenty pouch-young "tammar" wallabies (Macropus eugenii) (Liu, 2003). Outlined results of Liu demonstrate that developmental comparison between ABR and the focal responses from four auditory brainstem nuclei indicated that each ABR component may have a dominant contributor from the auditory brainstem, but there was no simple and exclusive association between ABR component and the auditory brainstem nuclei

In the past few decades, however, the role of ABR in assessing auditory functions in human being still remains controversial. Nekrassov \& Sitges (2003)'s study explores a possible connection between the reduced hearing sensitivity and certain abnormalities in ABR in generalized epilepsy. To this end, the effects of two convulsing agents, namely pentylenetetrazole and of 4-aminopyridine, on: (1) the cortical activity, (2) the hearing threshold and (3) the amplitudes and latencies of ABR waves evoked by a stimulus of high intensity $(100 \mathrm{~dB})$ were investigated in guinea pigs. The results show that changes in the activity of the lateral and the medial nuclei of the superior olivary complex accompany the hearing loss and the post-ictal epileptic cortical activity.

Increasing number of assessment tools, notably those relating to children throughout the world have created an urgent need for intensive investigations and strategies that clinicians and speech language therapists (SLTs) use to meet the highest standards and criteria of diagnosis. Research has shown that stacked-wave-V ABR requires a masking technique that may not be readily available to the clinician. Moreover, relatively high-level noise is required and may be annoying to the patient requires a masking technique that may not be readily available to the clinician. Since the only alternative choice was tone-bursts assessment tool, there was a need for more research to compare the merits and demerits of the two tools. Philibert, et al., (2003) have undertaken a comparative study between the two assessment tools. The overall objective of the researcher was "to explore a possible alternative approach, particularly one that might be both more accessible to the clinician, regardless of evoked potential test instrument used, and perhaps more acceptable to the patient." (Philibert, et al., 2003:p.2)

According to the research team, the observed results are promising for the use of the tonebursts assessment method as an alternative approach to ABR "stacking" and warrant further research and development of the tone- bursts method. A similar comparison like the one mentioned above was conducted by Szymańska, et al., (2008), but this time it was held between ABR thresholds and auditory steady-state response (ASSR) thresholds. According to 
the research team, auditory steady-state responses technique is not useful method in estimating of hearing threshold of young adults with normal hearing. Likewise, measurements of $\mathrm{ABR}$ and distortion-product otoacoustic emission were also compared to each other. According to Serpanos (2004), normative ABR and distortion-product otoacoustic emission templates of predicted loudness growth may have clinical application in site-of-lesion assessment or hearing aid fitting by distinguishing abnormal rates of loudness growth for individuals who cannot provide reliable behavioral measures.

Reconstructed waveforms are significantly affected by discrete wavelet transformation due to the positive/negative impacts of these waves and thus ABR outputs. The relationship between the two was examined by some neuropsycholinguists. The study of Wilson (2004) examined the relationship between ABR and its reconstructed waveforms following discrete wavelet transformation, and to comment on the resulting implications for ABR discrete wavelet transformation time-frequency analysis. Participants were 120 normal hearing subjects willing individuals range between 90, 70, 50, 30, 10 and 0 years old. Analysis found that reconstructed ABR discrete wavelet transformation waveforms can be used as valid time-frequency representations of the normal $\mathrm{ABR}$, but with some limitations. More specifically, the unexplained absence of a small number of reconstructed ABR discrete wavelet transformation waves in some subjects, probably resulting from "shift invariance" inherent to the discrete wavelet transformation process, needs to be addressed. As a matter of fact, such conclusions motivated other neuropsycholinguists to investigate the role played by imaging techniques in matters concerning measurements. The use of enhanced magnetic resonance imaging (MRI) as the standard to screen for small tumors is considered to be one of the most significant results due to the failure of standard ABR measures to detect small (\&lt; or $=1 \mathrm{~cm}$ ) acoustic tumors. An important conclusion drawn by Don, et al., (2005) is related to the stacked ABR that can be used, according to Don and his co-workers, as a sensitive, widely-available, cost-effective, and comfortable tool for screening small acoustic tumors. In order ensure the research validity and reliability; the researchers compared the stacked ABR amplitude to that of the standard ABR amplitude measurements. The research team found that "the amplitude of the stacked ABR wave V reflects more directly the total amount of activity initiated across the cochlea." (Don, et al., 2005: 279)

Overall, the above mentioned results provide strong support for investigating the validity and reliability of ABR. Neuropsycholinguists believe that in light of research findings, recommendations to improve the results of ABR could be made for new researchers, SLTs, programs' designers and other people in the field. The article of Johnson \& Brown (2005) presents information on various clinical studies that were conducted to dis/prove the validity and reliability of ABR. Again, the study aims at evaluating the accuracy with which ASSR and tone burst ABR thresholds predict behavioral thresholds, using a within-subjects design. ASSR and ABR thresholds were recorded in a group of 14 adults with normal hearing, 10 adults with flat, sensorineural hearing losses, and 10 adults with steeply sloping, high-frequency, sensorineural hearing losses. The researchers conclude that both ABR and the ASSR provided reasonably accurate predictions of behavioral threshold across the three subject groups. There was no evidence that the predictive accuracy of ABR evoked using 
Blackman-gated tone bursts differed from the predictive accuracy observed when linear-gated tone bursts were presented in conjunction with notched noise and vice versa.

Statistically, hearing capabilities vary from an infant to another. The aim of Marttila \& Karikoski (2006)'s study was to predict hearing level thresholds with click-evoked ABR and to study the residual hearing when ABR was absent. Armed with the results of 85 hearing-impaired children, the two researchers concluded to that the accuracy of ABR is reasonably ineffective, because it overestimates the hearing loss in moderate and severe impairments. The absence of ABR indicates a significant hearing loss which means that a lack of response to click stimuli does not directly suggest none-viable residual hearing. The development of ABR is subject to debate. Song, et al., (2006) studied such development to quantitatively assess its dependence on stimulus frequency and level. Responses were not observed to stimuli $>=16 \mathrm{kHz}$ on $\mathrm{P} 12$, however, the full range of responsive frequencies included in the study was observed by P14. Response thresholds were high on P12, exceeding $100 \mathrm{~dB}$ SPL for all stimuli tested. The findings indicate that slopes of wave I amplitude-and latency-level curves were significantly steeper than those of adults during the neonatal period and the outcome of input-output analyses, as well as frequency-specific maturational profiles, support developmental models in which function initially matures in the mid-frequency range and proceeds, simultaneously, in both apical and basal directions. Describing these complex relationships between latency level cures, slopes and wave, Song and her workmates have rightly observed: "In one group latency-level curves were relatively shallow, even adult like, whereas slopes were considerably steeper than those observed in adults in the other group. It is also notable that latency-level curves exhibiting relatively shallow, adult like slopes were generally associated with lower thresholds than those with steep slopes." (Song, et al., 2006: p.2249)

Again, Gorga, et al., (2006)'s study examines the accuracy with which click-evoked and tone burst-evoked ABR thresholds predict pure-tone audiometric thresholds. The in-depth observation of the ways in which pure-tone audiometric thresholds could be predicted helped the researchers to come up with important conclusions. Some of these conclusions are related to differences between ABR and behavioral threshold depended on behavioral threshold. This can be clearly seen with ABR thresholds overestimating behavioral threshold in cases of normal hearing and underestimating behavioral threshold in cases of hearing loss. Such results, according to Gorga and his co-workers, suggest that ABR thresholds can be used to predict pure-tone behavioral thresholds for a wide range of frequencies.

In Külekçi, et al., (2007)'s article, recent research is described in which age-equivalent norms for a $500 \mathrm{~Hz}$ logon evoked $\mathrm{ABR}$ could be obtained. The sample of the research was seventy-seven infants and children ranging from conceptional age of 40 weeks (term babies) to 4 years were tested with both click and tonal ABR without any risk factors for hearing loss. Külekçi, et al., (2007)'s results indicate that five hundred hertz of tonal ABR wave V latency did not mature until to 2-4 years old but, click ABR wave $V$ latency reached maturity at the same age range. Researchers have suggested that difficulties with temporal interactions or retrieval may be associated with age. Lavoie, et al., (2008)'s study assessed the changes in linear and nonlinear temporal interactions in the aging auditory brainstem of humans using 
maximum length sequence stimulation of 30 normal hearing females aged between 11 and 61years old. Results indicate that the linear and nonlinear components of the maximum length sequence -ABR could be more sensitive to changes occurring in the auditory system before any functionally significant changes in hearing status. The significance of such outcomes, according to the researchers, is that the nonlinear components of the auditory brainstem could be used as an investigative tool to assess early changes in the aging auditory brainstem in young middle-aged women.

Gaddam \& Ferraro (2008)'s study explores the effect of the improved sensitive ABR recordings on the hearing of the newborns via a procedure that utilizes the ear canal as a recording site. The two researchers compared the conventional recordings with ear canal recordings of ABR in 45 normally-hearing newborns that passed their newborn hearing screening. Results indicate that the amplitude of wave I is significantly larger and easier to identify when the ear canal is used as one of the recording sites in comparison to more conventional scalp (mastoid) recordings. Gaddam and his co-worker emphasized the importance of further research on the phenomenon under investigation. Such significance is a must for two reasons: "Firstly, develop a normative database that includes comparison of ABR component amplitudes in ear canal versus scalp recordings in newborns and infants. Secondly, further research is necessary to study the utility of the ear canal approach in recording cochlear receptor potentials in newborns and the possible clinical applications for doing this." (Gaddam \& Ferraro, 2008: p. 500)

During language production and comprehension, information about hearing properties is sometimes needed. While the decision about the significant role played by ABR in evaluating children's hearing capacities, Ribeiro \& Carvallo (2008)'s study was designed to investigate the feasibility of applying tone-ABRs in the nursery and neonatal intensive care unit, and to provide normative tone-ABR data from neonates. To that end, normative tone-ABR latency data were determined. Preterm neonates' ABRs showed significantly longer latencies than those of the full-term infants. Tone-ABR evaluation was found to be both feasible and reliable as a measure of auditory function in neonates. Regarding the ABR's amplitude-detection response correlational measurements, the two researchers clarified that such relationship was conditional. "In many cases of quiet, large amplitude ABRs with expected waveform morphology", Ribeiro \& Carvallo (2008) complete, "the visual detection of response in two channels added to the confidence in the response to eliminate the need for replication. Consequently, the overall test time was reduced which allowed time for the detection of responses at the $20 \mathrm{~dB}$ level." (Ribeiro \&Carvallo, 2008: p.28)

ABR is also thought to assess vestibular schwannomas and other cerebellopontine angle lesions. The aim of Grayeli, et al., (2008)'s study was to assess the value of ABR in the diagnosis of vestibular schwannomas and other cerebellopontine angle lesions. 676 solitary VS (548 operated on and 128 followed up) and 70 other CPA tumors (72\% meningiomas, $11 \%$ cholesteatomas, 3\% ependymomas, 15\% miscellaneous) managed between 1990 and 2001 completed a customized auditory test. Results of Grayeli and his colleagues reveal that ABRs were normal in $4.8 \%$ of VS. Association of normal ABR, vestibular caloric tests and audiometry (AAO-HNS class A) represented only $0.7 \%$ of VS. In other cerebellopontine 
angle lesions, ABR were normal in $15 \%$ of cases and the association of the three above-mentioned examinations was encountered in $10 \%$. However, in the latter cases the clinical examination showed an abnormality in all cases. Concerning the question of the role played by other electrophysiological techniques, the researchers made it clear that they can be used as complement assessment tools to ABR “....Other electrophysiological techniques have been designed to overcome several limitations of ABR." (Grayeli, et al., 2008: p. 1098)

There might be some arte-factual recording's risks as a result of an interaction between the simultaneous stimulation and recording. In order to avoid the risk of arte-factual recordings that might occur between the simultaneous stimulation and recording, and the similarities between the recording and the speech stimulus envelope, the study of Akhoun, et al., (2008) sought to systematically investigate the source of arte-factual contamination in speech ABR response. Results indicate that no responses were obtained from the deaf ear of unilaterally hearing impaired subjects, nor in the insert-out-of-the-ear condition in all the subjects, showing that speech ABR reflects the functioning of the auditory pathways.

In addition to arte-factual recording's risks, a threat might occur between the hearing pathways. It is for this reasons that researchers like Forti, et al., (2008) compare the pathway of hearing in both normal and adult patients. The purpose of Forti, et al., (2008)'s paper is to evaluate the hearing pathway in normal hearing adult patients. In order to achieve these goals, the researchers used ABRs to analyze absolute and inter-peak latencies in 19 adult DS patients aged 18-45 years whose pure tone audiometry test results indicated thresholds within normal limits, and 19 normal controls. The team's conclusions showed statistically significant gender-related differences in inter-peak interval III-V ( $p=0.015)$. The latencies of waves III and $\mathrm{V}$, and inter-peak intervals III-V and I-V, were significantly shortened in the patients under investigation than in the controls. According to Forti and his co-workers, such outcomes may be due to the smaller brain sizes and simpler afferent auditory pathways of subjects at hand.

Some researchers aim to investigate the effect of individual differences by comparing the results of a multiple/case study undertaken on a small group to another conducted on large number of participants under similar conditions to identify whether or not a remarkable significant differences could be observed. The article of Bush, et al., (2008) presents the results of a pilot study, including neuropsycholinguistic data, with patients underwent pure-tone audiometry followed by a determination of behavioral threshold and neuro-diagnostic threshold ABR in the normal ear, which was used as a control, and in the diseased ear. It attempts to determine if clinicians could increase the sensitivity of ABR testing in detecting small tumors. This means that replacing MRI with ABR was not a choice. In this regards, Bush and his workmates have rightly observed: "Our goal was not to attempt to replace MRI with ABR. Rather, we advocate the development of a cost-effective yet accurate algorithm for the diagnostic evaluation of patients with asymmetrical auditory symptoms." (Bush, et al., 2008: p.460).

The research method of Bush and his colleagues involved the use of a new ABR index that is based on threshold differences. Analysis of results revealed that all 7 patients had an 
abnormal ABR threshold difference, and 5 patients displayed abnormal traditional ABR indices. Furthermore, the mean difference between ABR and behavioral click thresholds was $41.4 \mathrm{~dB}$ in the diseased ears (with $\mathrm{ABR}$ threshold being higher than the click threshold) and $15.8 \mathrm{~dB}$ in the normal ears.

Bahmer, et al., (2008)'s study investigated the low-cost setup designed to perform an electrical-ABR as well as a conventional ABR for research purposes. Results from imaging techniques' analysis revealed that for ABR, only a personal computer with a soundcard, conventional system headphones, and an electroencephalography (EEG) pre-amplifier are necessary; for Electrical-ABR, in addition, an interface to the cochlea implant is required. Strictly, the capacity to assess and measure auditory performance is becoming increasingly important in the field of audiology. Inspired by the research of Bahmer and his co-workers, Dort, et al., (2009)'s research examined a new tool called power spectrum ABR as another tool in detecting patients at higher risk for auditory neuroma. The data used for his study are naturally collected. They are a mix of normal people and patients. In details, the participants were 53 subjects (19 subjects were normal controls and 34 subjects were patients with unilateral audio-vestibular symptoms). All subjects underwent complete auditory testing, standard ABR, stacked ABR, and power spectrum ABR. The 34 patients also underwent gadolinium enhanced MRI. The purpose of using such number was to in/validates the new assessment tool (power spectrum ABR). Early results of the study indicate that power spectrum ABR could be a valid and reliable method of identifying subgroups of patients with unilateral auditory dysfunction who would best benefit from MRI. Emphasizing the importance of such findings, Dort and his colleagues have rightly stated: "However, if ABR or some other electrophysiologic technique were more sensitive to the presence of small tumours, it could be a useful means of selecting higher-risk subpopulations of patients for higher-cost imaging studies. Such a prescreening strategy would reduce the number of negative MRI studies and reduce the demand for scarce MRI resources." (Dort, et al., 2009: p. 61)

In Swanepoel (2009)'s study, a description of ASSR and ABR thresholds for a group of infants and young children with various types and degrees of hearing loss is presented. A sample of 48 subjects (23 female) with a mean age of 2.8+/-1.9 years SD were assessed with a comprehensive test-battery and classified according to type and degree of hearing loss. The researcher summarizes many of the available methods and discusses their use with particular reference to the advantages and disadvantages of the two tools. Results show the reliability of verifying high frequency ASSR thresholds with a click-evoked ABR as an important cross-check in infants for whom behavioral audiometry may not be possible. Such outcome, according to Swanepoel "...indicated the best correlation between c-ABR and $2 \mathrm{kHz}$ followed by 4 and $1 \mathrm{kHz}$, respectively.” (Swanepoel, 2009: p. 218)

The neuropsycholinguistic perspective of whether or not cephalopods can detect sound frequencies above $400 \mathrm{~Hz}$ remains unanswered. In order to analyze and evaluate this issue, $\mathrm{Hu}$, et al., (2009) used ABR and found that auditory evoked potentials can be obtained in the frequency range 400 to $1500 \mathrm{~Hz}$ and 400 to $1000 \mathrm{~Hz}$, respectively. Furthermore, the thresholds of S. lessoniana were generally lower than those of O. vulgaris. Similarly, Van 
Maanen \& Stapells (2010)'s article presents a general introduction to the modern assessment tools and evaluative role. Various diagnostic methodologies are compared and contrasted. Particular attention is given to the theoretical background to the neuropsycholinguistic analysis. In the study of Van Maanen and Stapells, a comparison was conducted between multiple- ASSR and tone-ABR thresholds and assessed how well "normal" ASSR levels differentiate normal from elevated thresholds. Outlined results demonstrate that the multipleASSR and tone-ABR thresholds are strongly correlated, and the "normal" multiple- ASSR levels of 50,45, 40, and $40 \mathrm{~dB}$ HL correctly classified children as having "normal" or "elevated" thresholds. Summarizing the relationship between the two techniques, Van Maanen and Stapells have rightly noted: "Currently of much interest, the ASSR is typically detected using objective frequency domain statistical measures of response presence/absence, in contrast to the ABR where time-domain waveforms are usually visually identified by a clinician.” (Van Maanen \& Stapells, 2010: 536)

Clinical assessment tools are sometimes thought to be associated with concurrent difficulties in the area of medical fields. The study of Aimoni, et al., (2010) follows a group of 272 pediatric cases have been identified (544 ears). The purpose was to underline the role of $\mathrm{ABR}$ and electrocochleography in the definition of hearing loss in a selected group of children. In 19 of the 272 pediatric cases selected--38 ears (7\%), the results of threshold evaluation through ABR were uncertain. Outlined results show that ABR has to be considered the first choice in hearing assessment strategy, either for screening or for diagnosis in newborns as well as in non-collaborating children. Regarding electrocochleography, results indicate that it still may be considered a reliable diagnostic tool. Eighty (80) infants aged 0-6 months with normal hearing were administered the tone-pip ABR and ASSR thresholds in Qian et al., (2010)'s longitudinal study. Both were measured at octave frequencies from 250 to $8000 \mathrm{~Hz}$ bilaterally. Results of the research team suggest that with the maturational development, the conduction time of the auditory nerve decreases while the thresholds had no physiological changes within 0-6 months after birth. Comparing the tone-pip ABR with ASSR, the former had lower thresholds than the latter at $500-8000 \mathrm{~Hz}$, which indicates that the estimation of tone-pip ABR may be nearer to the actual hearing level of infants. Such findings, according to Qian and his co-workers, show "the threshold of tone-pip ABR at each frequency in infants within 6 months of life. The response thresholds of tone-pip ABR decreased as the frequency increased in different groups." (Qian, et al., 2010: p. 826)

Differences in the ways that ABR measurements help ensures accurate prescription for fitting infant hearing aids have long been of interest in the study of Bagatto (2010). Despite extensive theorizing, actual theoretical and empirical investigations have yet to converge on a coherent picture of the role played by ABR in estimating infants and children's hearing capabilities. As In this regards, Bagatto has rightly observed: "Infants younger than 6 months of age will have their hearing assessed using ABR procedures. If an adjustment is not already embedded into the system. ABR threshold estimates require adjustment prior to the calculation of the hearing aid prescription.” (Bagatto, 2010: p. 6)

Sinha \& Basavaraj (2010)'s paper investigates the speech evoked ABR to a consonant vowel stimulus. The total number of the participants of the study was thirty (30) subjects with 
normal hearing participated for the study. Findings demonstrate that understanding of speech evoked ABR has other applications both in research as well as in clinical purposes. As Sinha and his colleague have rightly stated: "The study described explicit method to record a speech evoked ABR to understand the neural basis of speech encoding." (Sinha \& Basavaraj, 2010: p. 398)

Such understanding, according to the two researchers, is of special importance, especially if one is interested in studying the central auditory system function.

The hearing capacity Profile of children was examined by Petoe, et al., (2010) with respect to gender and neurolinguistic variations. The purpose was to establish a variety of tools that are used to analyze the synchronicity of ABRs evoked by chirp- and click-stimuli at $40 \mathrm{~dB}$ HL. Auditory samples of 32 normal hearing subjects aged 18 to 55 years (mean $=24.8$ years, $\mathrm{SD}=7.1$ years) were gathered. They were analyzed according to type of speech disruption, speech rate, and capability of hearing disruptions. Statistical analysis found that the chirp-evoked ABRs exhibited less synchrony than the click-evoked ABRs in this study. The research team suggest that the temporal compensation offered by chirp stimuli is sufficient to increase neural recruitment (as measured by wave $\mathrm{V}$ amplitude), but that destructive phase interactions still exist along the cochlea partition, particularly in the low frequency portions of the cochlea where more latency jitter is expected. Neural synchrony measurements are not restricted to certain measurement tools. According to the team, "Other tools to quantify neural synchrony also exist, each with their own set of limitations." (Petoe, et al., 2010: 236)

The study of Coenraad, et al., (2010) used a total of 175 children (younger than 200 weeks post-conceptional age). The samples were referred for audiologic assessment with normal ABR results. The team made use of ABR parameters of normal hearing children between 2003 and 2008. The purpose of the study, according to Coenraad and his workmates, was to present a simple and powerful fitting model that describes age-dependent changes of ABR in a clinical population of normal hearing children. What motivated the team is the fact that "There was no fitting model to analyze ABR results in daily clinical practice reported in these studies.” (Coenraad, et al., 2010: p. 1532)

Outlined results show that such fitting model can be easily implemented to analyze ABR results of infants in daily clinical practice. ABR contains separate waves and signals. A dominant wave type in $\mathrm{ABR}$ is the wave $\mathrm{V}$ which is necessary in order to implement newborn hearing screening. In fact, some psychoneurolinguists believe that it is the most prominent and robust wave that has been used as indicator of hearing loss. In Arooj, et al., (2010)'s study, the instantaneous energy of ABR signal had been introduced as a marker to identify ABR waves. The study shows that the instantaneous energy of ABR can be used as a marker to identify ABR waves. Another study by Arooj and her colleagues had proposed a platform for fast hearing screening system. The team also recommends the best way to use instantaneous energy ABR signals. According to Arooj and her colleagues, such signals "can be used as marker in order to detect ABR waves." The team adds, "The performance of this method needs to be tested further." (Arooj, et al., 2010: p.820)

Yudhana, et al., (2010)'s study presents information on the turtle hearing capability by 
analyzing ABR spectral of two turtles ( 2 and 3 years accordingly). The study states that the turtle of 2 years old has peak power at $457 \mathrm{~Hz}$ in whole stimulus frequencies while the spectral amplitude is ranging $0.01-2.5 \%$ spectral. For the turtle of 3 years old, results show that it has peak power frequencies 50.78, 101.6, 152.3, 304.7, 355.5, 457, and $507.8 \mathrm{~Hz}$ respectively whereas the spectral amplitude is ranging $0.03-32.44 \%$ spectral. These results, according to Yudhana, et al., (2010) "show that turtle 3 years responds to the transmitted stimulus wider than turtle 2 years in frequency range and amplitude response." (Yudhana, et al., 2010: p. 194)

Clearly, there was a trend for ABR to have more accurate outcomes than any other hearing assessment tool in the field. To address such issue, Patterson, et al., (2010) used broad and white noise to characterize the system response and the digitized acoustic signal subsequently used to generate an acoustic inverse file capable of cancelling reverberations. Echo cancellation is nearly perfect, although location-specific. Results demonstrate that the spectral composition of the sound signal is not greatly affected. Describing the significance of the research findings, Patterson, et al., (2010) adds: "Our active echo cancellation procedure makes ABR experiments more readily doable outside of an anechoic environment. It therefore permits a wider range of organisms to be investigated, including aquatic organisms that are difficult (or impossible) to transport to shore side tanks, maintain in captivity, or both." (Patterson, et al., 2010: 862)

Importantly, the research team has successfully used the procedure during hearing studies of several fish species. Significant correlations were found between good diagnosis and correct use of the assessment tool and treatments. In order to get the best therapeutic outcomes, some researchers describe a corrective procedure that permits the clinician to obtain the estimated hearing level from ABR thresholds during infancy when immature responses are prevalent (Marcoux, 2011). The event-related potentials of ABR were investigated to study differences in the outcomes discrimination process. The results demonstrate that ABR assesses the competence and ensures excellence of its professional diplomats for clinical practice (adequate clinical response/ABR Writing Group, 2011).

National data were used to assess the impact of new ABR changes on pediatric radiology residency training in terms of the number, timing, availability, and funding of pediatric radiology rotations. An important conclusions that could be drawn from the study of Kollipara, et al., (2011) is that seven percent of residency programs plan to cut back the overall number of pediatric radiology core rotations and move them to earlier in training $(20 \%)$. Although $82 \%$ of programs said that they plan to provide more time in areas of interest, $57 \%$ said that it is very unlikely or impossible that residents could accumulate $\geq 12$ months in an area of interest. The primary objective of Dornan, et al., (2011)'s paper is to determine to what extent the factors contributing to the discrepancies between ABR thresholds play a role in the operating room and hearing outcomes obtained in the follow-up period. The study also explores the benefits and limitations of performing ABR in the operating room. To test this idea, a total of 116 patients were identified with complete records, including operating room-ABR results, medical examination and surgical procedure notes, and follow-up medical/audio-logical evaluation. Results indicate that in patients with 
middle ear effusion and tube placement, the average threshold difference between operating room-ABR and follow-up audio-logical evaluation was $9.7 \mathrm{~dB}( \pm 12.7)$, with highest discrepancy of $45 \mathrm{~dB}$. On the other hand, in patients with dry ears and/or no tube placement, the average threshold difference was only $3.8 \mathrm{~dB}( \pm 8.6)$. Specifically, the hearing thresholds estimated by $\mathrm{ABR}$ testing in the operating room can be elevated and follow-up hearing evaluation after tube placement is needed. These findings demonstrate that while ABR testing is valuable in the assessment of pediatric patients, especially for children who are difficult-to-test or have complicated medical conditions, caution should be taken when interpreting operating room-ABR results.

The study of Kuse, et al., (2011) presents the findings of a research study on evaluating changes in ABR in the course of auditory disturbance in rats induced by Kanamycin. Results demonstrate that some rats under the experiments showed an elevation of ABR threshold (15-40 dB SPL) and a decrease in amplitude of wave I and increase in amplitude of wave II at the same time. Commenting on the two results, the research team has rightly observed: "A few animals showed decreases in those of waves I and/or II. These changes may indicate a precursory phenomenon of the auditory disturbance. However, the increases in those amplitudes were considered to be a precursory phenomenon of the auditory disturbance." (Kuse, et al., 2011: p. 840)

Based on ABR examination, the team emphasize that the auditory disturbance of low-frequency to high-frequency range could be analyzed at an early date in detail. Such findings were later supported by some other studies (Ozaki, et al., 1996; Cunnick, et al., 2009; Alvarado, et al., 2012; Church, et al., 2012; Liu \& Chen, 2012).

Coenraad, et al., (2011) investigated ABR morphology. The purpose was to extend the current assessment system. Findings indicate that in $82 \%$ of the population, a typical "bow tie" response pattern was present as a sign of early auditory development. Furthermore, ABR morphology was also observed in the majority of the cases under investigation. In this regards, Coenraad and his colleagues have clearly stated: "ABR morphology was seen in 90\% of our population." (Coenraad, et al., 2011: p. 2249)

Using a meta-analysis design to examine the prevalence of a screening outcome pattern of ABR fail/otoacoustic emission pass (ABR-F/OAE-P), Berg, et al., (2011) found that Less than $1 \%$ of infants in well-infant nurseries had ABR-F/OAE-P screening outcomes as inpatients and none as outpatients. These results, according to Berg and other researchers, suggest that prevalence is low for infants cared for in WINs and use of OAE testing as a screening tool in WINs is not unreasonable. Examining whether or not neural encoding of speech features at the brain stem level is altered in the aging auditory system, in Vander, et al., (2011)'s study, speech-evoked ABRs were recorded using a synthetic 40-msec /da/ stimulus from both ears of participants in two groups: normal-hearing younger adults $(n=19)$ and normal-hearing older adults $(\mathrm{n}=18)$. The study also investigated the effect of minimal peripheral hearing loss on ABR evoked by speech stimuli and interactions with aging. Study's results support further investigation into the ability of the aging auditory system to encode temporal cues at the brain stem level, particularly the response to speech stimulus offset and 
its relationship to speech perception and temporal processing abilities.

The paper of Zhou, et al., (2012) targets assessment instruments used by SLTs in specific areas. Mainly, it reviews our experience of conducting ABR test on children in the operating room and discusses the benefits versus limitations of this practice. In the study 267 patients identified with usable data, including ABR results, medical and surgical notes, and follow-up evaluation. The main finding from the study reveal that hearing status successfully determined in all patients based on ABR results from the operating room. The most important result from the study indicates that multiple factors that may affect the outcomes of ABR in the operating room identified. Specifically, hearing loss in children with complicated medical issues can be accurately evaluated via ABR testing in the operating room. Unlike Zhou, et al., (2012)'s study, the article of Valderrama, et al., (2012) reviews some of the literature on differences in ABR's methodologies and discusses how these different methodologies may contribute to differences in ABR measurements. The researchers also suggest a methodology, which we will refer to as randomized stimulation and averaging that allows the recording of ABR at high stimulation rates using jittered stimuli. A comparison was conducted between the new method and the quasi-periodic sequence deconvolution and conventional stimulation methodologies. In conclusion, Valderrama and his workmates suggest that stimulation and averaging provides a quality in ABR recordings similar to that of quasi-periodic sequence deconvolution and conventional stimulation methodologies. Furthermore, the team believes that the feasibility of the randomized stimulation and averaging methodology is validated by an analysis of the morphology, amplitudes, and latencies of the most important waves in ABR recorded at high stimulation rates from eight normal hearing subjects.

Hornickel, et al., (2012)'s study examines the role of ABR measurements in assessing infants' hearing capacity. Speech evoked ABRs were collected from 26 typically-developing children (ages 8-13) at two time points separated by one year. Outlined results show that the consistency of response timing and spectral encoding found for the speech-evoked ABRs of typically-developing children suggests that the speech-evoked ABR may be a unique tool for research and clinical assessment of auditory function, particularly with respect to auditory-based communication skills. In their experimental study, Ruebhausen, et al., (2012) used a within-subject comparison and typical threshold criteria, to examine the effect of isoflurane and ketamine/xylazine on ABR thresholds for clicks and pure-tone stimuli extending from 8 to $32 \mathrm{kHz}$. The purpose of the research was to directly compare the effect of different anesthetics on ABR hearing threshold estimates. Ruebhausen and his colleagues emphasize that such highly significant threshold effect demonstrates a substantial difference between general anesthetics on auditory brainstem sensitivity.

Performance assessment techniques of hearing impairments can be influenced by a wide range of features of both task and participants. Naves, et al., (2012)'s study hypothesizes that there may be effects on the tool's assessment attributable to an interaction of variables such as the clinical environment, the age and/ or gender of the person under investigation. To this end, the researchers compared ABR manual/visual analysis provided by different examiners. As regards to corpus, ABR data were collected from 10 normal-hearing subjects ( 5 men and 5 women, from 20 to 52 years). A total of 160 data samples were analyzed and a pairwise 
comparison between four distinct examiners was executed. Based on such inputs, Naves and his colleagues drew an important conclusion. Describing these findings, the research team has rightly stated:

"Regression Analysis, together with the use of the Bootstrap for the assessment of the variability of the parameters of the liner model, is a suitable tool for detecting such discrepancies and their variability. From been employed for the characterization of the relationship between results obtained from distinct examiners and for different Jewett waves (I, II, III, IV and V). An important and innovative aspect of this research was the investigation of the variability of the discrepancies of the analyses of the examiners through the parameters ( $\beta 0$ and $\beta 1$ ) of a linear model using Bootstrap. We concluded that the parameter $\beta 1$ can be employed for checking the agreement between classifications of a particular Jewett wave.” (Naves, et al., 2012: pp. 522-523)

The purpose of Canale (2012)'s paper is to assess the reliability of Blackman windowed tone burst $\mathrm{ABR}$ as a predictor of hearing threshold at low frequencies. For this study, fifty-six subjects were divided in to three groups (normal hearing, conductive hearing loss, sensorineural hearing loss) after pure tone audiometry testing. Then they underwent tone burst ABR using Blackman windowed stimuli at $0.5 \mathrm{kHz}$ and $1 \mathrm{kHz}$. The authors compare the outcomes of the analysis with pure tone audiometry threshold. Mean threshold differences between pure tone audiometry and ABR ranged between $11 \mathrm{~dB}$ at $0.5 \mathrm{kHz}$ and $14 \mathrm{~dB}$ at 1 $\mathrm{kHz}$. ABR threshold was worse than pure tone audiometry in each but 2 cases. Mean discrepancy between the two thresholds was about $20 \mathrm{~dB}$ in normal hearing, reducing in presence of hearing loss, without any differences in conductive and sensorineural cases.

The study of Turchetta, et al., (2012) reveals how a population derived from a newborn hearing screening protocol, some aspects of maturation of the auditory pathways in the first months after birth, and the possible repercussions on early treatment are evaluated by means of ABR. An important outcome of the study is that the auditory system might not be completely developed at birth. More importantly, this auditory system, according to the research team, might require some months to complete; hence any early clinical approach should consider the possibility of an overtreatment, and any therapeutic strategy should only be considered once the diagnosis is certain and definitive. Mühlenberg \& Schade (2012)'s study used 32 patients with a severe hearing loss at low-frequencies and 28 patients with normal hearing. The purpose was to evaluate behavioral and ABR thresholds to a low-chirp respectively a $500 \mathrm{~Hz}$ tone presented in notched noise masking. Outlined results of Mühlenberg and his workmate show a significant equivalence between low-chirp-ABR and pure-tone audiometric. ABR to a low-chirp stimulus seems to be rather precise in contrast to the notched-noise-500 Hz-evoked-ABR in order to diagnose a low-frequency-hearing loss. Öztürk \&Genç (2012)'s study examined the effects of ABR against infants' hearing capability in order to establish age-related maturational changes for infants aged 0-6 months. 180 subjects from 0 months to 6 months of age were measured by ABR. Results are discussed in terms of age-related changes in adutory processing and context use in infants. According to Öztürk and his workmate, Knowledge on ABR characteristics within first six months of life will enable clinicians to discriminate normal situations from pathologic ones in diagnosing 
hearing loss for the infant population.

ABR is more vulnerable to clinical assessment than many other devices, notably when evaluating infants who are more susceptible to hearing loss. Hatton, et al., (2012) hypothesized that the test performance of the bone-conduction tone-evoked ABR in infants with hearing deficit is not successful. Results demonstrate that the "normal" bone-conduction-ABR levels accurately differentiated normal versus elevated cochlear sensitivity (accuracy: $98 \%$ for $2000 \mathrm{~Hz} ; 98 \%$ for $500 \mathrm{~Hz}$ ). According to the researchers, these findings further support the use of $\mathrm{BC}$ tone ABR for diagnostic ABR testing. Lima, et al., (2012) investigates the influence of the ketamine/xylazine anesthetic on ABR latency values in adult gerbils. In the study, ABRs of 12 adult gerbils injected with the anesthetic were collected on three consecutive days, or a total of six collections, namely: pre-collection and A, $\mathrm{B}, \mathrm{C}, \mathrm{D}$, and $\mathrm{E}$ collections. The research team concluded to saying that the use of ketamine/xylazine increases the latency of the $\mathrm{V}$ wave of ABR after several doses injected into adult gerbils. Clinicians, according to Lima and his friends, should consider the use of this substance in the assessment of ABR.

Auditory system behavior refers to the systematic functions performed by behavior in certain times. Such "systematicity" is of special importance when examining ABR. In order to evaluate the inter-examiner agreement and variability in the manual classification of ABR, Naves, et al., (2012) studied 160 ABR data samples. The study aims at evaluating the inter-examiner agreement and variability in the manual classification of ABR. The results of the study quantify the inter-examiner agreement and variability of the manual analysis of ABR data, and they also allow for the determination of different patterns of manual ABR analysis. Some addressed the clinical statement of both ACR and ABR and emphasized their role in credentialing and privileging of radiologists for diagnostic nuclear medicine, including multimodality hybrid imaging (Gannon, 2012).

Auditory diagnostic tools are known to aggregate in the medical field, notably that of speech language pathology. Debate among SLTs exists on whether the available devices, especially old ones present an additional risk for children. Destigter, et al., (2012)'s study recommends new structures as new developments to be added to ABR. According to the research team, the new ABR examination structure gives programs an opportunity to evaluate this practice. The article of Silva, et al., (2012) highlights various results and findings about the roles played by ABR in auditory issues. One of these findings and outcomes is that ABR play a critical role in down-regulating hypoxia-induced pulmonary hypertension. The study of Henry, et al., (2012) considered the impact of noninvasive ABR in both clinical and research environment is very important. The team discussed the correlation between ABR characteristics and more direct measures of cochlear function by recording ABRs and auditory nerve single-unit responses in seven chinchillas with noise-induced hearing loss. Results indicate that ABR thresholds and wave I amplitude provide useful estimates of cochlear sensitivity. Furthermore, comparisons of ABR wave I latency to normative data at the same sensation level may prove useful for detecting and characterizing loss of cochlear frequency selectivity.

The reduction in repetition is a lexical property determining measurements among ABR tests. 
Acir, et al., (2013) used 488 ABR responses for creating two different data sets. The first set is created conventionally by ensemble averaging of 1,024 single trials for each ABR pattern. The second set is obtained from the first estimated 64 single trials of the same records for each ABR. While the obtained results contribute to the practical ABR usage in clinics, Acir and his colleagues add, the great significance of it arises from the reduction in repetitions via estimation of ABRs. Experimental studies have indicated that ABR's results in matters concerning infants' accurate diagnosis differ from a country to another. Longitudinal studies have corroborated this difference by showing patterns differences in terms of hearing capacities, although no detailed study has been performed. The study of Li, et al., (2013) implemented 111 preterm (27-36weeks GA) and 92 term (37-41weeks GA) infants who were administered to tests at 6 weeks. Results indicate that there were no overall differences between term and preterm groups in ABRs. Furthermore, ABRs in preterm and term infants were similar at 6weeks (corrected age if preterm), but males had less advanced ABRs than females.

Lohr, et al., (2013)'s study examines whether or not ABR can measure the brain responses of birds. To address these questions, three woodpeckers were estimated by ABR. Results show that two (sometime three) prominent peaks occurring within $10 \mathrm{~ms}$ of stimulus onset. These results, according to the research team, suggest that woodpecker thresholds may be lower than those of domesticated birds, while similar to those of wild birds. The notched noise method (An involved effective procedure for measuring frequency resolution and auditory filter shapes in both human and animal models of hearing) in hearing measurements' processing is connected by several dorsal and ventral tracts, but the functional roles of the different tracts are not well understood. To test the importance of this method in the measurements of hearing processing, Lina (2013) combined relatively non-invasive ABR's measurements and the notched noise method to estimate auditory filters in normal-hearing mice at center frequencies of $8,11.2$, and $16 \mathrm{kHz}$. ABR notched noise procedure provides a fast alternative to estimating frequency selectivity in mice that is well-suited to high through-put or time-sensitive screening.

\subsection{Aims}

Reliability and validity of ABR measurements for Arab Children have not been investigated yet. This research is important to better understanding of the effects played by ABR measurements on diagnosing Arab children's auditory functions. Additionally, the study provides additional evidence on whether or not $\mathrm{ABR}$ is reliable and valid by answering the following questions:

1. Can ABR measure auditory functions of Arab infants and children with hearing disorders the way it is designed for?

2. Are there any significant differences between the results of the first ABR measurement test and the second?

\subsection{Methodology}

This study is conducted in the age group of 0 month $(\mathrm{N}=30)$ to 3 year old Arab children $(\mathrm{N}=30)$ at $\mathrm{Al}$ Khars hospital, Al Aḥsa'a, Kingdom of Saudi Arabia. Subjects under 
investigation were divided into two groups: Group 1 includes infants ( $0-40$ weeks) and group 2 includes children (40 weeks - 3years) of both sexes. ABR pre-and-posttest of measurement is administered within two weeks to both groups. As it is mentioned in the aims of this research, the purpose is to examine the validity and reliability of ABR in Arab infants and children. To that end, the time between the first and second tests was only one week. Outcomes of the two tests were neuropsycholinguistically and statistically analyzed in light of neuropsycholinguistics.

\section{Analysis}

\subsection{Validity \& Reliability of ABR for Infants' Measurements}

ABR, like most of other large-scale international clinical assessments, presents its measurement frameworks in multidimensional subscales. To fulfill the requirements of this multidimensional measurement framework, this experimental research deployed a new perspective to in/validates this measurement tool.

As for the subjects, as it is mentioned in the methodology of this research, they were divided into two groups: The first group contains infants between 0-10 months while the second group contains children between 10 months- 3 years. Both groups have undergone ABR investigation. To start with, the pre-test administered to the first group, a comprehensive investigation of the auditory functions of the infants shows that 3 of them, namely, Subject 9 (3 days old), subject 27 (two weeks old) and subject 29 (7 weeks old) have serious hearing problems that may develop (if not well treated) a total hearing loss. Other infants vary from partial auditory problems $(\mathrm{N}=8)$ to no significant hearing problems at all $(\mathrm{N}=19)$. Table 1 summarizes the results of the infants pre-test. Consider:

Table 1. Infants' ABR pre-test outcomes

\begin{tabular}{|c|c|}
\hline Subject No. & ABR pre-test outcomes \\
\hline Subject 1 & 9 \\
\hline Subject 2 & 7 \\
\hline Subject 3 & 9 \\
\hline Subject 4 & 8 \\
\hline Subject 5 & 10 \\
\hline Subject 6 & 8 \\
\hline Subject 7 & 7 \\
\hline Subject 8 & 7 \\
\hline Subject 9 & 2 \\
\hline Subject 10 & 8 \\
\hline Subject 11 & 9 \\
\hline Subject 12 & 9 \\
\hline Subject 13 & 9 \\
\hline Subject 14 & 7 \\
\hline Subject 15 & 9 \\
\hline
\end{tabular}




\begin{tabular}{|c|c|}
\hline Subject 16 & 8 \\
\hline Subject 17 & 9 \\
\hline Subject 18 & 7 \\
\hline Subject 19 19 & 9 \\
\hline Subject 20 & 10 \\
\hline Subject 21 & 9 \\
\hline Subject 22 & 10 \\
\hline Subject 23 & 9 \\
\hline Subject 24 & 8 \\
\hline Subject 25 & 7 \\
\hline Subject 26 & 8 \\
\hline Subject 27 & 3 \\
\hline Subject 28 & 9 \\
\hline Subject 29 & 1 \\
\hline Subject 30 & 9 \\
\hline Total subjects in question & 30 \\
\hline Total of intact subjects & 19 \\
\hline Total of subjects with partial auditory problems & 8 \\
\hline Total of subjects with serious auditory problem. & 3 \\
\hline
\end{tabular}

Clearly, in Table 1, ABR measured hearing capabilities of the infants. The purpose of administering this test as well as of the current study is to evaluate infants' auditory ability. In details, the first objective is to screen infants' hearing. The second aim is to estimate auditory threshold of the infants' under investigation. Intraoperative monitoring is another objective of the pre-test. On the top of that, ABR ought to determine not only the type of hearing loss, but also its degree of seriousness. Detection of newborns' auditory nerve and brainstem lesion is also another important objective of the present study. Having the test administered, results were reviewed in view of a tripled-scale designed by the researchers. (Scores between $0-5=$ serious case, scores between 6-8 = partial auditory problems, and scores between 9-10= no problem at all). Based on the above mentioned scale, ABR descriptive statistic results show that nineteen (19) of the subjects under investigation are nearly intact. In other words, they do not have any of the above mentioned auditory problems. Whereas eight (8) of the subjects registered partial auditory problems, three (3) of them scored no problem at all. No disease has been recognized in the test other than the hearing problems revealed by ABR in the infants at hand. Evidently, this means that the test examined what it was supposed to test. In clearer, terms, such outcomes prove the validity of the test.

Strictly, this study is an experimental investigation to determine not only the validity of ABR, but also to show whether or not it is reliable. In order to dis/ prove this research characteristic, the researchers have undertaken another test after a week of the pre-test. Standard statistical analyses were employed to compare the outcomes of the pre-test with those of the post-test. Significant differences were found in some of the subjects in question. 
The following table summarized the results of the post-test. Consider:

Table 2. Infants' ABR posttest outcomes

\begin{tabular}{|c|c|}
\hline Subject No. & ABR post-test outcomes \\
\hline Subject 1 & 10 \\
\hline Subject 2 & 7 \\
\hline Subject 3 & 10 \\
\hline Subject 4 & 7 \\
\hline Subject 5 & 9 \\
\hline Subject 6 & 7 \\
\hline Subject 7 & 8 \\
\hline Subject 8 & 8 \\
\hline Subject 9 & 1 \\
\hline Subject 10 & 7 \\
\hline Subject 11 & 9 \\
\hline Subject 12 & 10 \\
\hline Subject 13 & 9 \\
\hline Subject 14 & 8 \\
\hline Subject 15 & 10 \\
\hline Subject 16 & 8 \\
\hline Subject 17 & 9 \\
\hline Subject 18 & 8 \\
\hline Subject 19 & 9 \\
\hline Subject 20 & 9 \\
\hline Subject 21 & 10 \\
\hline Subject 22 & 9 \\
\hline Subject 23 & 10 \\
\hline Subject 24 & 7 \\
\hline Subject 25 & 7 \\
\hline Subject 26 & 7 \\
\hline Subject 27 & 2 \\
\hline Subject 28 & 10 \\
\hline Subject 29 & 2 \\
\hline Subject 30 & 9 \\
\hline Total subjects in question & 30 \\
\hline Total of intact subjects & 19 \\
\hline Total of subjects with partial auditory problems & 8 \\
\hline Total of subjects with serious auditory problem & 3 \\
\hline
\end{tabular}

As can be clearly seen in Table 2, nearly the measurements in all subjects are the same with 


\section{MInstitute Macrothink $^{m}$}

Journal for the Study of English Linguistics

ISSN 2329-7034

2013, Vol. 1, No. 2

no remarkable differences. Out of the total number of the subjects under investigation, the measurements of nineteen (19) subjects of them (the same subjects in the pre-test) did not change. The same thing applies to the rest of subjects regardless the degree of seriousness their auditory hearing suffer from. The following table, however, sumps up the differences between the outcomes of both the pre-and-posttest. Compare:

Table 3. Infants' ABR pre-and-posttest: Comparison

\begin{tabular}{|c|c|c|}
\hline Subject No. & Pre-test ABR Outcomes & Post-test ABR Outcomes \\
\hline Subject 1 & 9 & 10 \\
\hline Subject 2 & 7 & 7 \\
\hline Subject 3 & 9 & 10 \\
\hline Subject 4 & 8 & 7 \\
\hline Subject 5 & 10 & 9 \\
\hline Subject 6 & 8 & 7 \\
\hline Subject 7 & 7 & 8 \\
\hline Subject 8 & 7 & 8 \\
\hline Subject 9 & 2 & 1 \\
\hline Subject 10 & 8 & 7 \\
\hline Subject 11 & 9 & 9 \\
\hline Subject 12 & 9 & 10 \\
\hline Subject 13 & 9 & 9 \\
\hline Subject 14 & 7 & 8 \\
\hline Subject 15 & 9 & 10 \\
\hline Subject 16 & 8 & 8 \\
\hline Subject 17 & 9 & 9 \\
\hline Subject 18 & 7 & 8 \\
\hline Subject 19 & 9 & 9 \\
\hline Subject 20 & 10 & 9 \\
\hline Subject 21 & 9 & 10 \\
\hline Subject 22 & 10 & 9 \\
\hline Subject 23 & 9 & 10 \\
\hline Subject 24 & 8 & 7 \\
\hline Subject 25 & 7 & 7 \\
\hline Subject 26 & 8 & 7 \\
\hline Subject 27 & 3 & 2 \\
\hline Subject 28 & 9 & 10 \\
\hline Subject 29 & 1 & 2 \\
\hline Subject 30 & 9 & 9 \\
\hline \multicolumn{2}{|c|}{ Total subjects in question } & 30 \\
\hline \multicolumn{2}{|c|}{ Total of intact subjects } & 19 \\
\hline
\end{tabular}




\begin{tabular}{|c|c|}
\hline $\begin{array}{c}\text { Total of subjects with } \\
\text { partial auditory problems }\end{array}$ & 8 \\
\hline $\begin{array}{c}\text { Total of subjects with } \\
\text { serious auditory problems }\end{array}$ & 3 \\
\hline
\end{tabular}

It is obviously found in Table 3 that slightly significant differences have been registered between the two tests. In details, the subjects in the posttest have generally a higher score measurements than in the pre-test (I score). Measurements of intact subjects were more likely to register the highest significant differences than those of partial or serious hearing problems and this can be clearly observed in view of the two tests' outcomes ( 7 and 3 respectively). Although it was anticipated that infants' posttest measurements would score higher in matters concerning partial hearing problems, this was not borne out by the findings and indeed they scored lower on the tripled scale (4/5). Infants' ABR pre-test measurements did score higher in matters relating to serious auditory problems and this can be clearly seen in the scores of the scores of subjects 9,27 and 29 ( 2 and 1,3 and 2, and 1 and 2 respectively). All in all, when accounting for such analysis, it can be said that no remarkable or significant differences between the pre-and-posttests which means that ABR is reliable.

\subsection{Validity \& Reliability for ABR Children's Measurements}

Measuring functional capabilities of infants and/ or children's hearing is a major part of ABR's functions. Nearly all possible hearing problems that might occur in the period between 10 months and 3 years have been considered. The recognition that environmental factors may influence auditory capabilities has given rise to studies examining the relationship between neighborhood conditions and risk for hearing disorders. Since the purpose of the study is to in/ validate whether or not ABR is reliable, influence like time length, neighborhood and parents effects have been avoided. The period of time in this research did not exceed a week. Generally speaking, it is observed that many studies drew a great attention to the role of ABR in measuring infants' hearing capabilities, but older children have yet to receive the needed research attention. To that end, the current study paid a close attention to children as it did to the infants. Thirty (30) children ranges between 10 months and 3 years have been used in this study. Children's auditory capabilities have been measured by ABR. The following table illustrates the results of this pre-test measurement. Consider:

Table 4. Children's ABR pre-test outcomes

\begin{tabular}{|c|c|}
\hline Subject No. & ABR pre-test outcomes \\
\hline Subject 1 & 10 \\
\hline Subject 2 & 8 \\
\hline Subject 3 & 10 \\
\hline Subject 4 & 8 \\
\hline Subject 5 & 10 \\
\hline Subject 6 & 7 \\
\hline Subject 7 & 8 \\
\hline Subject 8 & 8 \\
\hline
\end{tabular}




\begin{tabular}{|c|c|}
\hline Subject 9 & 10 \\
\hline Subject 10 & 7 \\
\hline Subject 11 & 10 \\
\hline Subject 12 & 1 \\
\hline Subject 13 & 10 \\
\hline Subject 14 & 6 \\
\hline Subject 15 & 9 \\
\hline Subject 16 & 9 \\
\hline Subject 17 & 10 \\
\hline Subject 18 & 8 \\
\hline Subject 19 & 9 \\
\hline Subject 20 & 9 \\
\hline Subject 21 & 9 \\
\hline Subject 22 & 10 \\
\hline Subject 23 & 10 \\
\hline Subject 24 & 7 \\
\hline Subject 25 & 7 \\
\hline Subject 26 & 7 \\
\hline Subject 27 & 7 \\
\hline Subject 28 & 10 \\
\hline Subject 29 & 9 \\
\hline Subject 30 & 10 \\
\hline Total subjects in question & 30 \\
\hline Total of intact subjects & 17 \\
\hline $\begin{array}{l}\text { Total of subjects with partial audito } \\
\text { problems }\end{array}$ & 12 \\
\hline $\begin{array}{l}\text { Total of subjects with serious audito } \\
\text { problems }\end{array}$ & 1 \\
\hline
\end{tabular}

ABR children's pre-test sought an understanding of ABR measurements on the subjects under investigation in terms of who suffers from hearing disorders and to what extent he/ she suffers. However, ABR's pre-test measurements show that out of the thirty subjects at hand, more than half of them are not affected. Subjects who belong to this category are those whose scores of measurements range between nine (9) and ten (10). The rest of the subjects as it is clearly observed in the above mentioned table, suffer either from a partial hearing disorders (12 subjects) and these subjects are given the scores between 6- 8, or complete/ serious hearing loss ( 1 subject) who is given the scores between 1-5. Having the pre-test administered, the second step was to administer the posttest which has been conducted a week later. The following table explains in numbers the outcomes of the children ABR posttest measurements. Consider: 
Table 5. Children's ABR posttest outcomes

\begin{tabular}{|c|c|}
\hline Subject No. & ABR posttest outcomes \\
\hline Subject 1 & 10 \\
\hline Subject 2 & 7 \\
\hline Subject 3 & 9 \\
\hline Subject 4 & 8 \\
\hline Subject 5 & 9 \\
\hline Subject 6 & 8 \\
\hline Subject 7 & 8 \\
\hline Subject 8 & 8 \\
\hline Subject 9 & 10 \\
\hline Subject 10 & 6 \\
\hline Subject 11 & 9 \\
\hline Subject 12 & 2 \\
\hline Subject 13 & 10 \\
\hline Subject 14 & 7 \\
\hline Subject 15 & 10 \\
\hline Subject 16 & 9 \\
\hline Subject 17 & 9 \\
\hline Subject 18 & 8 \\
\hline Subject 19 & 10 \\
\hline Subject 20 & 9 \\
\hline Subject 21 & 10 \\
\hline Subject 22 & 10 \\
\hline Subject 23 & 10 \\
\hline Subject 24 & 8 \\
\hline Subject 25 & 7 \\
\hline Subject 26 & 7 \\
\hline Subject 27 & 8 \\
\hline Subject 28 & 10 \\
\hline Subject 29 & 9 \\
\hline Subject 30 & 9 \\
\hline Total subjects in question & 30 \\
\hline Total of intact subjects & 17 \\
\hline Total of subjects with partial auditory problen & 12 \\
\hline $\begin{array}{l}\text { Total of subjects with serious audito } \\
\text { problems }\end{array}$ & 1 \\
\hline
\end{tabular}

As it can be seen in the above mentioned table, there are some differences between the same scales in comparison to pre-test. Again, such differences cannot be described as significant 


\section{$\Lambda$ Macrothink}

Journal for the Study of English Linguistics

ISSN 2329-7034

2013, Vol. 1, No. 2

differences because they do not make big differences in the total score of the measurement. Strictly, the present research is guided by the tripled scale which means the score does not make a remarkable difference if it is within the same range of the scale. For example, if the pre-test measurement score is 7 and in the posttest it is 8 , there is no significant difference because both of the two scores belong to the same range (6-9). However, the difference becomes important when it differs from one range of scale to another (e., 3 and 8). In that sense, it can be said that the results of the two tests' measurements are, generally speaking, similar. A distinction ought to be made between the two tests. Such distinction will be clearly understood in light of the following table. Compare:

Table 6. Children's ABR pre-and-posttest: Comparison

\begin{tabular}{|c|c|c|}
\hline Subject No. & ABR pre-test outcomes & ABR posttest outcomes \\
\hline Subject 1 & 10 & 10 \\
\hline Subject 2 & 8 & 7 \\
\hline Subject 3 & 10 & 9 \\
\hline Subject 4 & 8 & 8 \\
\hline Subject 5 & 10 & 9 \\
\hline Subject 6 & 7 & 8 \\
\hline Subject 7 & 8 & 8 \\
\hline Subject 8 & 8 & 8 \\
\hline Subject 9 & 10 & 10 \\
\hline Subject 10 & 7 & 6 \\
\hline Subject 11 & 10 & 9 \\
\hline Subject 12 & 1 & 2 \\
\hline Subject 13 & 10 & 10 \\
\hline Subject 14 & 6 & 7 \\
\hline Subject 15 & 9 & 10 \\
\hline Subject 16 & 9 & 9 \\
\hline Subject 17 & 10 & 9 \\
\hline Subject 18 & 8 & 8 \\
\hline Subject 19 & 9 & 10 \\
\hline Subject 20 & 9 & 9 \\
\hline Subject 21 & 9 & 10 \\
\hline Subject 22 & 10 & 10 \\
\hline Subject 23 & 10 & 10 \\
\hline Subject 24 & 7 & 8 \\
\hline Subject 25 & 7 & 7 \\
\hline Subject 26 & 7 & 7 \\
\hline Subject 27 & 7 & 8 \\
\hline Subject 28 & 10 & 10 \\
\hline
\end{tabular}




\begin{tabular}{|c|c|}
\hline Subject 29 & 9 \\
\hline Subject 30 & 9 \\
\hline Total subjects in question & 30 \\
\hline Total of intact subjects & 17 \\
\hline $\begin{array}{c}\text { Total of subjects with partia } \\
\text { auditory problems }\end{array}$ & 12 \\
\hline $\begin{array}{c}\text { Total of subjects with seriou } \\
\text { auditory problems }\end{array}$ & 1 \\
\hline
\end{tabular}

Analyses are performed in Table 6 within data come from Tables 4 and 5. Rapid look at the measurements on the two columns (pre-and-posttest) reveal a slightly difference (1 score for posttest) which makes the result almost the same. Among 17 unaffected subjects, only two pre-test measurements were statistically significant as they go in the expected direction. What is expected is that the measurements increase as times passes where the child's immunity grew stronger.

Partial hearing problems were associated with fewer score among children's ABR pre-test measurements in comparison to ABR posttest's measurements wherein they registered higher ones (I score) and it had no effect on the total number of scores since the registered score was in the same tripled scale (i.e., 6-8). Likewise, the increase in the ABR pre-test measurements of the children with serious hearing problems was not significantly related to the age because the child is not below 40 weeks so that we can say that he/ she prone to diseases. The researchers believe that is normal and it does not make a significant difference since it is only one score. Again, the scores in the two tests are almost similar which indicate that the ABR measurements for children are reliable.

\section{Conclusion}

\subsection{Infants and Children's pre-and-posttest ABR Measurements: Validity \& Reliability}

Differences in hearing capabilities and functions may diminish with age. We investigated the validity and reliability of ABR among Arab infants and children taking into consideration such differences. Cross-sectional data were available from sixty cohorts (Infants $=30$ and children= 30). Meta-analyses indicated that overall perfect hearing capability favored children. Normally, performance declined with age, but not with immunity that increases with the passage of time.

Infants' ABR pre-and-posttest results show that there was a quite big difference between those who are, clinically speaking, intact or unaffected (3/7). Statistically, these differences in the rate of decline in healthy infants' pre-test are not significant, because both results of pre-and-posttest belong to the same band of tripled scale (i.e., 9-10). Intact children's ABR pre-and-posttest differences were also examined. Two scores for the measurements of children in the pre-test were statistically observed. Again, this does not make them significant due to that they belong to the same band or category in the scale assigned by the researchers. Consistent with expectations, infants in the ABR pre-test perceived more partial hearing disorders when they first measured ( 5 scores) and less than this number in the ABR posttest's measurements (4 scores). As it can be seen, the effects were larger for the infants in the pre-test than in the posttest and that may be accounted for because of their age where they are 
prone to infectious diseases. Relative to the two tests, there is no significant difference between the two scores since they belong to the same band of scale (6-8). In pre-and-posttest analyses that only included children with partial hearing problems, the difference was positive and significantly associated with the posttest measurements where four children scored partial problems in the test compared with only two subjects in the pre-test measurements. The results will only be considered as statistically remarkable if the two scores' difference belong to another categorical band in the tripled scale. Since they belong to the same band (6-8), it remains insignificant.

However, infants with poor hearing showed trends for change in the scores of the pre-and-posttest. Whereas they scored better in the pre-test (1 more score), the measurement in the posttest was not the same. In clearer terms, they did not reach the same score in the posttest measurement. The reverse was seen in children where one more score has been registered in the posttest. Again, the score be it more or less does not make important differences because they belong to the same band of categorization.

In general, with a few notable exceptions, there are no scores' effects on the general outcome of the study which means that the ABR measurements for both Arab infants and children are similar. This means in any way that ABR proved to be valid and reliable. Such conclusion is better explained in light of the following figure:

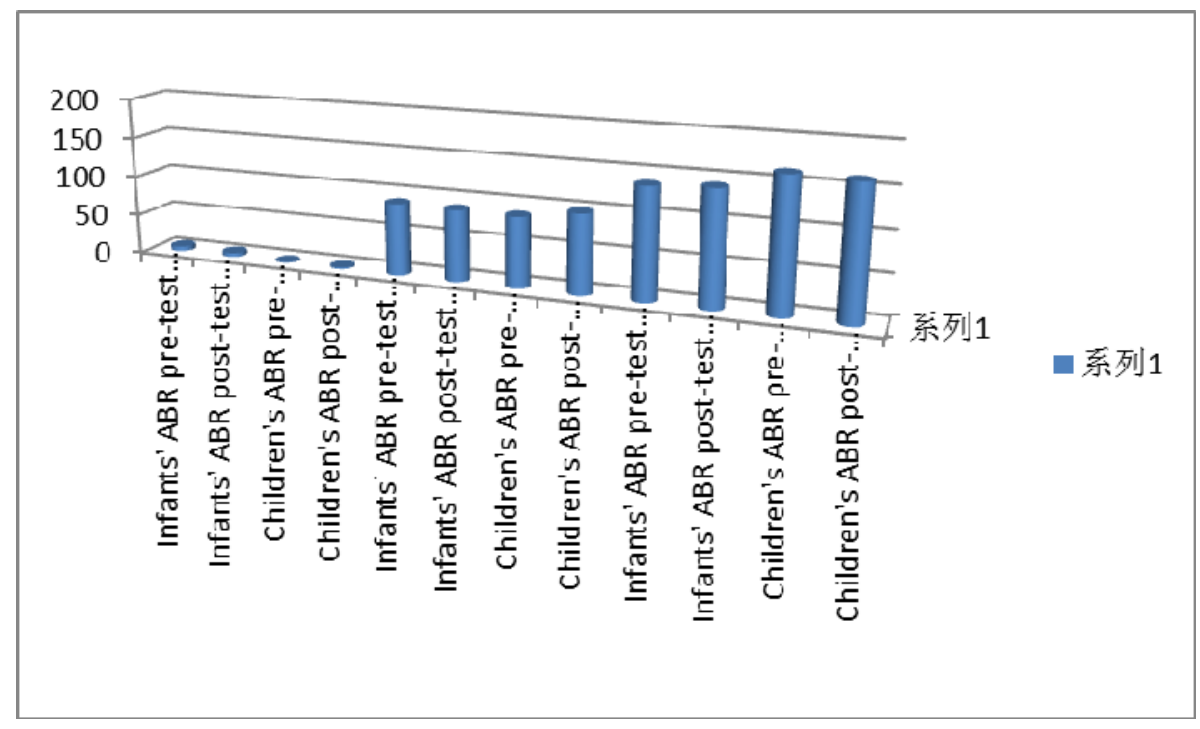

Figure 1. Comparison between Infants and children's pre-and-posttest ABR measurements: Validity \& Reliability

As can be seen in Figure 1, the measurements of ABR for both infants' pre-and-posttest are almost the same and the same thing applies to the degree of their hearing problems' seriousness (no hearing problems at all, partial hearing problems, and serious hearing problems). Likewise, children's ABR measurements for both pre-and-posttests are almost the same, not only in the case of complete hearing loss, but also in the cases of partial and serious 
hearing problems. Undoubtedly, this means that ABR is valid and reliable when measuring Arab children.

\subsection{ABR Measurements: Comparative Outcomes}

This is the first report of the ABR measurements for the hearing capabilities of both Arab infants and children. As it is mentioned somewhere in this research, the aim of the study is to prove whether or not ABR measurements of both Arab infants and children are valid and reliable. A correlational analysis was used to determine the strength of magnitude of the relationship between subjects' performance and the degree of their hearing capacity's seriousness. Descriptive statistics were used to compare the scores of the subjects from both groups (infants' group and children's group) on a tripled scale scores $(0-5=$ serious hearing problems, $6-8=$ partial hearing problems, and $9-10=$ no hearing problems at all). Findings of the study indicated that infants and children's ABR measurements are similar in both pre-and-posttests which demonstrate that $\mathrm{ABR}$ is both valid and reliable.

The effect on the scores of the two groups did not vary significantly by levels of categorical bands on the tripled scale and neuropsycholinguistic resources except for the few significant cross-level interactions. Such findings support Murray's study (1988). Subjects' ages were associated with the degree of hearing problems, and no significant difference was registered among all participants neither in their pre-test nor in their posttest ABR measurements. This conclusion agrees with the findings of many other studies (Kawarai, et al., 1999; Johnson \& Brown, 2005; Hornickel, et al., 2012; Silva, et al., 2012; Öztürk \&Genç, 2012).

As a matter of fact, it would be difficult to generalize this study across all the age classes. Practical implications should be taken into account. The study would help clinicians devise strategies for both the infants and children and across different age groups, in order to establish relationships with their brands. Social implications should be also mentioned. The study provides insights into the audio-logical performance of infants and children with respect to their interactions with $\mathrm{ABR}$ measurements. It throws light on the change in hearing capabilities' measurements with increasing age and how the basis for relationships formation varies. The paper combines age as well as gender differences and the role of affect and cognition in the clinical context. Ultimately, it is hoped that the results of this study will help guide the development of ABR for the development of measurements for all children.

\section{References}

Acır, N., Erkan, Y., \& Bahtiyar, Y. (2013). Auditory brainstem response classification for threshold detection using estimated evoked potential data: comparison with ensemble averaged data. Neural Computing \& Applications, 22(5), 859-867.

Aimoni, C., Ciorba, A., Bovo, R., Trevisi, P., Busi, M., \& Martini, A. (2010). Hearing threshold assessment in young children with electrocochleography (EcochG) and auditory brainstem responses (ABR): experience at the University Hospital of Ferrara. Auris, Nasus, Larynx, 37(5), 553-557. http://dx.doi.org/10.1016/j.anl.2010.02.002. 
Akhoun, I., Moulin, A., Jeanvoine, A., Ménard, M., Buret, F., Vollaire, C., \& Thai-Van, H. (2008). Speech auditory brainstem response (speech ABR) characteristics depending on recording conditions, and hearing status: An experimental parametric study. Journal Of Neuroscience Methods, 175(2), 196-205. http://dx.doi.org/10.1016/j.jneumeth.2008.07.026.

Alvarado, J., Fuentes-Santamaría, V., Jareño-Flores, T., Blanco, J., \& Juiz, J. (2012). Normal variations in the morphology of auditory brainstem response (ABR) waveforms: a study in Wistar rats. Neuroscience Research, 302-311. http://dx.doi.org/10.1016/j.neures.2012.05.001.

Arooj, A., Muhamed, M., Salleh, S., \& Omar, M. (2010). Use of instantaneous energy of ABR signals for fast detection of wave V. Journal Of Biomedical Science \& Engineering, 3(8), 816-821. http://dx.doi.org/10.4236/jbise.2010.38109.

Bagatto, M. (2010). Fitting infant hearing aids: ABR measurements help ensure accurate prescription. ASHA Leader, 15(7), 5-6.

Bahmer, A., Peter, O., \& Baumann, U. (2008). Recording of electrically evoked auditory brainstem responses (E-ABR) with an integrated stimulus generator in Matlab. Journal Of Neuroscience Methods, 173(2), 306-314. http://dx.doi.org/10.1016/j.jneumeth.2008.06.012.

Beck, D. L., Speidel, D. P., \& Petrak, M. (2007). Auditory Steady-State Response (ASSR): A Beginner's Guide. The Hearing Review. 2007; 14(12), 34-37.

Berg, A. L., Prieve, B. A., Serpanos, Y. C., \& Wheaton, M. A. (2011). Hearing Screening in a Well-Infant Nursery: Profile of Automated ABR-Fail/OAE-Pass. Pediatrics, 127(2), 269-275. http://dx.doi.org/http://dx.doi.org/10.1542/peds.2010-0676.

Block, K., Amie, A., Chery, J., Gina, N., Peggy, A., Deborah, W., \& Sara, H. (1993). "Definitions of Communication Disorders and Variations", Ad Hoc Committee on Service Delivery in the Schools. ASHA, 13-14. http://dx.doi.org/10.1044/policy. RP1993-00208, retrieved 2010-08-07.

Bush, M. L., Jones, R. O., \& Shinn, J. B. (2008). Auditory brainstem response threshold differences in patients with vestibular schwannoma: A new diagnostic index. ENT: Ear, Nose \& Throat Journal, 87(8), 458-462.

Canale, A., Dagna, F., Lacilla, M., Piumetto, E., \& Albera, R. (2012). Relationship between pure tone audiometry and tone burst auditory brainstem response at low frequencies gated with Blackman window. European Archives Of Oto-Rhino-Laryngology, 269(3), 781-785. http://dx.doi.org/10.1007/s00405-011-1723-7.

Church, M. W., Hotra, J. W., Holmes, P. A., Anumba, J. I., Jackson, D. A., \& Adams, B. R. (2012). Auditory Brainstem Response (ABR) Abnormalities Across the Life Span of Rats Prenatally Exposed to Alcohol. Alcoholism: Clinical \& Experimental Research, 36(1), 83-96. http://dx.doi.org/http://dx.doi.org/10.1111/j.1530-0277.2011.01594.x.

Coenraad, S., Immerzeel, T. v., Hoeve, L., \& Goedegebure, A. (2010). Fitting model of ABR age dependency in a clinical population of normal hearing children. European Archives Of 
Oto-Rhino-Laryngology: Official Journal Of The European Federation

OfOto-Rhino-Laryngology: Official Journal of The European Federation Of $\begin{array}{lll}\text { Oto-Rhino-Laryngological Societies } & \text { (EUFOS), }\end{array}$ http://dx.doi.org/10.1007/s00405-010-1291-2.

Coenraad, S., Toll, M., Hoeve, H., \& Goedegebure, A. (2011). Auditory brainstem response morphology and analysis in very preterm neonatal intensive care unit infants. The Laryngoscope, 121(10), 2245-2249. http://dx.doi.org/10.1002/lary.22140.

Cunnick, J., Schmidhuber, S., Chen, G., Yu, M., Yi, S., Cho, Y., \& Heisterkamp, N. (2009). $\mathrm{Bcr}$ and $\mathrm{Abr}$ cooperate in negatively regulating acute inflammatory responses. Molecular And Cellular Biology, 29(21), 5742-5750. http://dx.doi.org/10.1128/MCB.00357-09.

Destigter, K., Mainiero, M., Janower, M., \& Resnik, C. (2012). Resident Clinical Duties While Preparing for the ABR Core Examination: Position Statement of the Association of Program Directors in Radiology. Journal Of The American College Of Radiology, 9(11), 832-834. http://dx.doi.org/http://dx.doi.org/10.1016/j.jacr.2012.05.012.

Don, M., Kwong, B., Tanaka, C., Brackmann, D., \& Nelson, R. (2005). The stacked ABR: a sensitive and specific screening tool for detecting small acoustic tumors. Audiology \& Neuro-Otology, 10(5), 274-290.

Dornan, B., Fligor, B., Whittemore, K., \& Zhou, G. (2011). Pediatric hearing assessment by auditory brainstem response in the operating room. International Journal Of Pediatric Otorhinolaryngology, 75(7), 935-938. http://dx.doi.org/10.1016/j.ijporl.2011.04.010.

Dort, J., Cook, E., Watson, C., Shaw, G., Brown, D., \& Eggermont, J. (2009). Power spectrum auditory brainstem response: novel approach to the evaluation of patients with unilateral auditory symptoms. Journal Of Otolaryngology - Head \& Neck Surgery = Le Journal D'oto-Rhino-Laryngologie Et De Chirurgie Cervico-Faciale, 38(1), 59-66.

Eggermont, J. J., Burkard, R. F., \& Manuel, D. (2007). Auditory evoked potentials: basic principles and clinical application. Hagerstwon, MD: Lippincott Williams \& Wilkins, p. 3. ISBN 0-7817-5756-8. OCLC 70051359.

Forti, S., Amadeo, C., Fagnani, E., Filipponi, E., Pignataro, L., Cesarani, A., \& Ambrosetti, U. (2008). Auditory brainstem responses (ABR) in normal hearing adult subjects with Down's syndrome. Brain Research, 123358-62. http://dx.doi.org/10.1016/j.brainres.2008.07.078.

Gaddam, A., \& Ferraro, J. (2008). ABR recordings in newborns using an ear canal electrode. International Journal Of Audiology, 47(8), 499-504.

Gannon, J. (2012). Re: "ACR/ABR clinical statement on credentialing and privileging of radiologists for diagnostic nuclear medicine, including multimodality hybrid imaging". Journal Of The American College Of Radiology: JACR, 9(1), 82-83. http://dx.doi.org/10.1016/j.jacr.2011.10.016.

Garnham, J., Cope, Y., Durst, C., McCormick, B., \& Mason, S. (2000). Assessment of aided ABR thresholds before cochlear implantation. British Journal Of Audiology, 34(5), 267-278. 
Gorga, M., Johnson, T., Kaminski, J., Beauchaine, K., Garner, C., \& Neely, S. (2006). Using a combination of click- and tone burst-evoked auditory brain stem response measurements to estimate pure-tone thresholds. Ear \& Hearing (01960202), 27(1), 60-74.

Grayeli, A., Refass, A., Smail, M., Elgarem, H., Kalamarides, M., Bouccara, D., \& Sterkers, O. (2008). Diagnostic value of auditory brainstem responses in cerebellopontine angle tumours. Acta Oto-Laryngologica, 128(10), 1096-1100. http://dx.doi.org/10.1080/00016480701881803.

Hatton, J., Janssen, R., \& Stapells, D. (2012). Auditory brainstem responses to bone-conducted brief tones in young children with conductive or sensorineural hearing loss. International Of Journal Otolaryngology, 2012284864. http://dx.doi.org/10.1155/2012/284864.

Henry, K. S., Kale, S., Scheidt, R. E., \& Heinz, M. G. (2011). Auditory brainstem responses predict auditory nerve fiber thresholds and frequency selectivity in hearing impaired chinchillas. Hearing Research, 280(1/2), 236-244. http://dx.doi.org/10.1016/j.heares.2011.06.002.

Hornickel, J., Knowles, E., \& Kraus, N. (2012). Test-retest consistency of speech-evoked auditory brainstem responses in typically-developing children. Hearing Research, 284(1/2), 52-58. http://dx.doi.org/10.1016/j.heares.2011.12.005.

Hu, M., Yan, H., Chung, W., Shiao, J., \& Hwang, P. (2009). Acoustically evoked potentials in two cephalopods inferred using the auditory brainstem response (ABR) approach. Comparative Biochemistry And Physiology. Part A, Molecular \& Integrative Physiology, 153(3), 278-283. http://dx.doi.org/10.1016/j.cbpa.2009.02.040.

Johnson, T., \& Brown, C. (2005). Threshold prediction using the auditory steady-state response and the tone burst auditory brain stem response: a within-subject comparison. Ear \& Hearing (01960202), 26(6), 559-576.

Kawarai, H., Nishizaki, K., Fukuda, S., Akagi, S., Inokuchi, I., Gunduz, M., \& Masuda, Y. (1999). Hearing immaturity found by ABR and its clinical impact on otoneurological evaluation. Acta Oto-Laryngologica. Supplementum, 5406-11.

Kollipara, R., Rentas, K., \& Lowe, L. (2011). Impact of ABR changes on pediatric radiology residency training. Journal of The American College Of Radiology: JACR, 8(11), 804-808. http://dx.doi.org/10.1016/j.jacr.2011.04.013.

Külekçi, S. S., Terlemez, Ş. Ş., Çiprut, A. A., \& Akdaş, F. F. (2007). 500Hz logon versus click ABR maturation.International . Journal Of Pediatric Otorhinolaryngology, 71(5), 775-779. http://dx.doi.org/10.1016/j.ijporl.2007.01.016.

Kuse, H., Ogawa, T., Nakamura, N., Nakayama, Y., Nakakarumai, A., Komori, C., \& Tamura, K. (2011). Changes in auditory brainstem response (ABR) in Kanamycin-induced auditory disturbance model rats. Journal Of Toxicological Sciences, 36(6), 835-841. 
Lavoie, B. A., Mehta, R. R., \& Thornton, A. D. (2008). Linear and nonlinear changes in the auditory brainstem response of aging humans. Clinical Neurophysiology, 119(4), 772-785. http://dx.doi.org/10.1016/j.clinph.2007.12.004.

Lei, S., McGee, J., \& Walsh, E. J. (2006). Frequency- and level-dependent changes in auditory brainstem responses (ABRs) in developing mice. Journal Of The Acoustical Society Of America, 119(4), 2242-2257. http://dx.doi.org/10.1121/1.2180533.

Li, M., Zhu, L., Mai, X., Shao, J., Lozoff, B., \& Zhao, Z. (2013). Sex and gestational age effects on auditory brainstem responses in preterm and term infants. Early Human Development, 89(1), 43-48. http://dx.doi.org/10.1016/j.earlhumdev.2012.07.012.

Lima, J., Ariga, S., Velasco, I., \& Schochat, E. (2012). Effect of the ketamine/xylazine anesthetic on the auditory brainstem response of adult gerbils. Brazilian Journal Of Medical And Biological Research = Revista Brasileira De Pesquisas Médicas EBiológicas / Sociedade Brasileira De Biofisica ... [Et Al.], 45(12), 1244-1247.

Lina, I. A., \& Lauer, A. M. (2013). Rapid measurement of auditory filter shape in mice using the auditory brainstem response and notched noise. Hearing Research, 29873-79. http://dx.doi.org/10.1016/j.heares.2013.01.002.

Liu, G. (2003). Functional development of the auditory brainstem in the tammar wallaby (Macropus eugenii): the superior olivary complex and its relationship with the auditory brainstem response (ABR). Hearing Research, 175(1/2), 152.

Liu, X., \& Chen, L. (2012). Auditory brainstem response as a possible objective indicator for salicylate-induced tinnitus in rats. Brain Research, 148588-94. http://dx.doi.org/10.1016/j.brainres.2012.04.048.

Lohr, B., Brittan-Powell, E., \& Dooling, R. (2013). Auditory brainstem responses and auditory thresholds in woodpeckers. The Journal Of The Acoustical Society Of America, 133(1), 337-342. http://dx.doi.org/10.1121/1.4770255.

Marcoux, A. M. (2011). The Manipulation of Auditory Brain Stem Response Data to Predict the Behavioral Audiogram during Infancy. Seminars In Hearing, 32(2), 156-162. http://dx.doi.org/http://dx.doi.org/10.1055/s-0031-1277237.

Marttila, T., \& Karikoski, J. (2006). Comparison between audiometric and ABR thresholds in children Contradictory findings. European Archives Of Oto-Rhino-Laryngology: Official Journal Of The European Federation Of Oto-Rhino-Laryngological Societies (EUFOS): Affiliated With The German Society For Oto-Rhino-Laryngology - Head And Neck Surgery, 263(5), 399-403.

Mühlenberg, L., \& Schade, G. (2012). [A comparison of low-chirp- and notched-noise-evoked auditory brainstem response]. Laryngo- Rhino- Otologie, 91(8), 500-504. http://dx.doi.org/10.1055/s-0031-1291330. 
Murray, A. D. (1988). Newborn Auditory Brainstem Evoked Responses (ABRs): Longitudinal Correlates in the First Year. Child Development, 59(6), 1542. http://dx.doi.org/10.1111/1467-8624.ep8591097.

Naves, K. P., Pereira, A. A., Nasuto, S. J., Russo, I. C., \& Andrade, A. O. (2012). Analysis of the variability of auditory brainstem response components through linear regression. Journal Of Biomedical Science \& Engineering, 5(9), 517-525. http://dx.doi.org/10.4236/jbise.2012.59064.

Naves, K., Pereira, A. A., Nasuto, S. J., Russo, I., \& Andrade, A. O. (2012). Assessment of inter-examiner agreement and variability in the manual classification of auditory brainstem response. Biomedical Engineering Online, 11(1), 86-95. http://dx.doi.org/10.1186/1475-925X-11-86.

Nekrassov, V., \& Sitges, M. (2003). Effects of pentylenetetrazole and 4-aminopyridine on the auditory brainstem response (ABR) and on the hearing sensitivity in the guinea pig in vivo. Epilepsy Research, 53(3), 245. http://dx.doi.org/10.1016/S0920-1211(03)00031-7.

Niedermeyer, E., \& da-Silva, F. L. (2004). Electroencephalography: Basic Principles, Clinical Applications, and Related Fields. Lippincot Williams \& Wilkins, p. 4. ISBN 0-7817-5126-8.

Ozaki, H., Kurata, K., Horinouchi, A., \& Ando, T. (1996). Auditory brainstem response (ABR) and effects of furosemide on ABR in conscious F344 rats. The Journal Of Toxicological Sciences, 21(3), 167-175.

Öztürk, B., \& Gen, C. (2012). Maturation of auditory brainstem responses in babies from brith to 6 months of age [Turkish]. Turkiye Klinikleri Journal Of Medical Sciences, 32(3), 677-686. http://dx.doi.org/http://dx.doi.org/10.5336/medsci.2011-24809.

Patterson, M. R., Horodysky, A. Z., Deffenbaugh, B. W., \& Brill, R. W. (2010). Using active echo cancellation to minimize stimulus reverberations during hearing studies conducted with the auditory brain response (ABR) technique. Journal Of Biomedical Science \& Engineering, 3(9), 861-867. http://dx.doi.org/10.4236/jbise.2010.39116.

Petoe, M. A., Bradley, A. P., \& Wilson, W. J. (2010). On chirp stimuli and neural synchrony in the suprathreshold auditory brainstem response. Journal Of The Acoustical Society Of America, 128(1), 235-246. http://dx.doi.org/10.1121/1.3436527.

Philibert, B., Durrant, J., Ferber-Viart, C., Duclaux, R., Veuillet, E., \& Collet, L. (2003). Stacked tone-burst-evoked auditory brainstem response (ABR): preliminary findings. International Journal Of Audiology, 42(2), 71-81.

Purdy, S., \& Abbas, P. (2002). ABR thresholds to tonebursts gated with Blackman and linear windows in adults with high-frequency sensorineural hearing loss. Ear \& Hearing (01960202), 23(4), 358-368.

Qian, L., Yi, W., Xingqi, L., Yinsheng, C., Wenying, N., Lili, X., \& Yinghui, L. (2010). Development of tone-pip auditory brainstem responses and auditory steady-state responses in 
infants aged 0-6 months. Acta Oto-Laryngologica, 130(7), 824-830. http://dx.doi.org/http://dx.doi.org/10.3109/00016480903471069.

Ribeiro, F., \& Carvallo, R. (2008). Tone-evoked ABR in full-term and preterm neonates with normal hearing. International Journal Of Audiology, 47(1), 21-29.

Ruebhausen, M., Brozoski, T., \& Bauer, C. (2012). A comparison of the effects of isoflurane and ketamine anesthesia on auditory brainstem response (ABR) thresholds in rats. Hearing Research, 287(1-2), 25-29. http://dx.doi.org/10.1016/j.heares.2012.04.005.

Serpanos, Y. (2004). ABR and DPOAE Indices of Normal Loudness in Children and Adults. Journal Of The American Academy Of Audiology, 15(8), 555-565.

Sheil, W. (2012). "Magnetic Resonance Imaging (MRI Scan)". Medicine, 3.

Silva, N. M., Pereira, L., Poloni, E. S., \& Currat, M. (2012). Lack of Bcr and Abr Promotes Hypoxia-Induced Pulmonary Hypertension in Mice. Plos ONE, 7(11), 1-12.

Sinha, S., \& Basavaraj, V. (2010). Speech Evoked Auditory Brainstem Responses: A New Tool to Study Brainstem Encoding of Speech Sounds. Indian Journal Of Otolaryngology \& Head \& Neck Surgery, 62(4), 395-399. http://dx.doi.org/10.1007/s12070-010-0100-y.

Swanepoel, D., \& Ebrahim, S. (2009). Auditory steady-state response and auditory brainstem response thresholds in children. European Archives Of Oto-Rhino-Laryngology: Official Journal Of The European Federation Of Oto-Rhino-Laryngological Societies (EUFO)): Affiliated With The German Society For Oto-Rhino-Laryngology - Head And Neck Surgery, 266(2), 213-219. http://dx.doi.org/10.1007/s00405-008-0738-1.

Szymańska, A., Gryczyński, M., \& Pajor, A. (2008). [The estimation of behavioral audiograms, auditory brainstem response (ABR) thresholds and auditory steady-state response (ASSR) thresholds of young adults with normal hearing]. Otolaryngologia Polska $\begin{array}{llll}\text { The Polish } & \text { Otolaryngology, } & \text { 62(6), } & \text { 735-739. }\end{array}$ http://dx.doi.org/10.1016/S0030-6657(08)70350-0.

Turchetta, R., Orlando, M., Cammeresi, M., Altissimi, G., Celani, T., Mazzei, F., \& Cianfrone, G. (2012). Modifications of auditory brainstem responses (ABR): observations in full-term and pre-term newborns. Journal Of Maternal-Fetal \& Neonatal Medicine, 25(8), 1342-1347. http://dx.doi.org/10.3109/14767058.2011.634457.

Uzuka, Y., Furuta, T., Yamaoka, M., Ohnishi, T., Tsubone, H., \& Sugano, S. (1996). Threshold changes in auditory brainstem response (ABR) due to the administration of kanamycin in dogs. Experimental Animals / Japanese Association For Laboratory Animal Science, 45(4), 325-331.

Valderrama, J., Alvarez, I., de-la-Torre, A., Segura, J., Sainz, M., \& Vargas, J. (2012). Recording of auditory brainstem response at high stimulation rates using randomized stimulation and averaging. The Journal Of The Acoustical Society Of America, 132(6), 3856-3865. http://dx.doi.org/10.1121/1.4764511. 
Vander-Werff, K., \& Burns, K. (2011). Brain stem responses to speech in younger and older adults. Ear And Hearing,32(2), 168-180. http://dx.doi.org/10.1097/AUD.0b013e3181f534b5.

Van-Maanen, A., \& Stapells, D. R. (2010). Multiple-ASSR Thresholds in Infants and Young Children with Hearing Loss. Journal Of The American Academy Of Audiology, 21(8), 535-545. http://dx.doi.org/10.3766/jaaa.21.8.5.

Various-Authors, A. (2011). ACR/ABR clinical statement on credentialing and privileging of radiologists for diagnostic nuclear medicine, including multimodality hybrid imaging. Journal of the American College of Radiology, 8(9), 617-621.

Werner, L., Folsom, R., Mancl, L., \& Syapin, C. (2001). Human auditory brainstem response to temporal gaps in noise. Journal Of Speech, Language \& Hearing Research, 44(4), 737-750.

Wilson, W. J. (2004). The relationship between the auditory brain-stem response and its reconstructed waveforms following discrete wavelet transformation. Clinical Neurophysiology, 115(5), 1129. http://dx.doi.org/10.1016/j.clinph.2003.11.019.

Yudhana, A., Din, J., Abdullah, S., \& Hassan, R. (2010). Turtle Hearing Capability Based on ABR Signal Assessment. Telkomnika, 8(2), 187-194.

Zhou, G., Dornan, B., \& Hinchion, W. (2012). Clinical experience of auditory brainstem response testing on pediatric patients in the operating room. International Journal Of Otolaryngology, 2012350437, 2-7. http://dx.doi.org/10.1155/2012/350437.

\section{Glossary}

ABR (Auditory Brainstem Response) It is a neurologic test of auditory brainstem function in response to auditory (click) stimuli. First described by Jewett and Williston in 1971, ABR audiometry is the most common application of auditory evoked responses. The resulting recording is a series of vertex positive waves of which I through $\mathrm{V}$ are evaluated. These waves, labeled with roman numerals in Jewett and Williston convention, occur in the first 10 milliseconds after onset of an auditory stimulus. ABR is a helpful tool in determining a child's ability to hear. The test uses a special computer to measure the way the child's hearing nerve responds to different sounds (Eggermont, et al., 2007: p. 3).

ASSR (Auditory Steady-State Response) It is an objective test used for evaluation of hearing ability in children too young for traditional audiometric testing. ABR is an electro-physiologic response to rapid auditory stimuli. The goal of ASSR is to create an estimated audiogram from which questions regarding hearing, hearing loss, and aural rehabilitation can be answered. Most children are referred for ASSR after a newborn hearing screen in the hospital indicates the possibility of hearing loss. ASSR is similar to the ABR in some respects. For example, ASSR and ABR record bioelectric activity from electrodes arranged in similar recording arrays. ASSR and ABR are both auditory evoked potentials. ASSR and ABR use acoustic stimuli delivered through inserts (preferably). ASSR and ABR have important differences, too. Rather than depending on amplitude and latency, ASSR across a spectrum, rather than peak detection across a time versus amplitude waveform. 
ASSR is evoked using repeated sound stimuli presented at a high repetition rate, whereas ABR is evoked using brief sounds presented at a relatively low repetition rate (Beck, et al., 2007: pp.34-37).

EEG (Electroencephalography) It is a neurological test that uses an electronic monitoring device to measure and record electrical activity in the brain. EEG is the recording of electrical activity along the scalp. It measures voltage fluctuations resulting from ionic current flows within the neurons of the brain. The EEG is a key tool in the diagnosis and management of epilepsy and other seizure disorders (Niedermeyer \& da Silva, 2004: p. 4)

MRI (Magnetic Resonance Imaging) = It is a test that uses a magnetic field and pulses of radio wave energy to make pictures of organs and structures inside the body (Sheil, 2012, P. 3).

SLTs (Speech-Language Therapists) $=$ They are specialized in communication disorders as well as swallowing disorders. They are also called Speech Pathologists (Block et al., 1993, P. 23)

\section{Copyright Disclaimer}

Copyright reserved by the author(s).

This article is an open-access article distributed under the terms and conditions of the Creative Commons Attribution license (http://creativecommons.org/licenses/by/3.0/). 